L'irradiation d'un solide

par des particules de haute énergie est à l'origine de modifications importantes,

souvent défavorables, de ses propriétés d'usage : on parle d'endommagement.

\section{Dans cet article, on décrit}

brièvement les mécanismes

d'interaction entre les neutrons

rapides et la matière, qui se

traduisent par la formation

de cascades de déplacements

atomiques, mais aussi par des

réactions de transmutation

nucléaire qui modifient la

composition chimique.

Les endommagements

macroscopiques qui en

résultent (en particulier

gonflement, durcissement

et fragilisation) dépendent

fortement de la structure

cristalline et de la composition

chimique du matériau irradié

et limitent sa durée d'utilisation dans les réacteurs nucléaires

de fission ou de fusion.

\title{
Endommagement des alliages métalliques par les neutrons rapides
}

Jean-Louis Boutard (jean-louis.boutard@cea.fr)

Cabinet du Haut-Commissaire à l'énergie atomique, CEA/Saclay, 91191 Gif-sur-Yvette Cedex

\section{Interaction des neutrons rapides avec la matière [1]}

Les neutrons issus de la fission de l'uranium ou de la fusion du deutérium et du tritium, ont des énergies cinétiques respectives de $\sim 2$ et de $14 \mathrm{MeV}$, et sont appelés "rapides". Les neutrons étant électriquement neutres, pénètrent profondément dans la plupart des solides et interagissent avec les noyaux atomiques via les forces nucléaires. Cette interaction avec la matière donne lieu à des réactions nucléaires inélastiques qui induisent des transmutations ${ }^{(a)}$, et à des chocs élastiques qui déplacent les atomes de leurs sites. En effet, l'énergie transférée par un neutron rapide lors d'une collision avec un noyau est largement supérieure à l'énergie nécessaire pour déplacer de façon permanente tout atome de son site (à titre d'exemple, cette dernière est de $40 \mathrm{eV}$ dans le fer, alors que l'énergie moyenne transférée par un neutron de $1 \mathrm{MeV}$ à un atome de fer est de $35 \mathrm{keV}$ ). Ces premiers atomes frappés (PKA en anglais : Primary Knocked-on Atoms) perdent ensuite leur énergie cinétique par interaction inélastique avec les électrons (pouvoir d'arrêt électronique), et par chocs élastiques nucléaires successifs avec d'autres noyaux de la cible, dont un certain nombre sont à leur tour déplacés.

\section{Cascades de déplacements atomiques}

Vers la fin de leur parcours, les atomes frappés provoquent des cascades de déplacements atomiques (fig. 1), dont une modélisation par dynamique moléculaire est donnée sur la figure 2. Un déplacement atomique est formé d'une lacune, qui est la place laissée libre par l'atome éjecté de son site cristallin, et d'un atome interstitiel (en abrégé, "un interstitiel ») qui s'insère entre les atomes de la structure cristalline initiale.

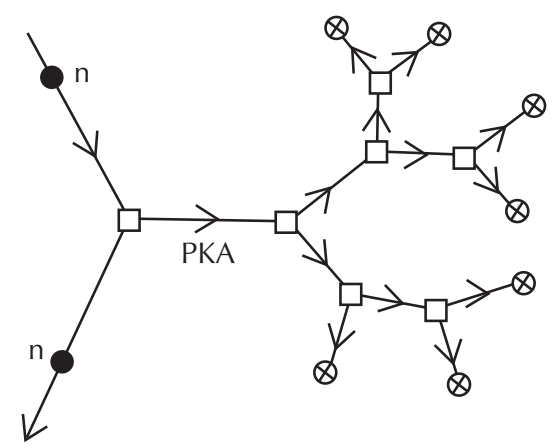

1. Représentation schématique d'une cascade de déplacements.

$n$ : neutron $\quad \square$ : lacune $\otimes$ : interstitiel

Une cascade de déplacements est un événement localisé dans l'espace et le temps, et qui affecte fortement la structure cristalline du solide irradié. La taille typique d'une cascade est de l'ordre de $10 \mathrm{~nm}$. Pendant la première phase, dite balistique, qui dure quelques 0,1 ps, il y a création d'un grand nombre de défauts dont la majorité s'éliminent (par recombinaison ou migration) pendant les 1 à 10 ps suivantes, ce qui correspond à environ 10 à 100 vibrations du réseau cristallin, en faisant l'hypothèse d'une fréquence de Debye de $10^{13} \mathrm{~s}^{-1}$. À la fin de cette seconde phase, dite de recuit, il reste des amas de lacunes au centre de ce qui fut la cascade, et des interstitiels en périphérie ; ces amas résiduels de défauts, très difficiles à mettre en évidence expérimentalement, n'ont pu être identifiés à ce jour que dans le tungstène par microscopie ionique à émission de champ [2].

La dose reçue est quantifiée par le nombre de déplacements par atome (dpa). Par exemple, on dit que le matériau a reçu une dose de 100 dpa si chaque atome a été déplacé en moyenne 100 fois. 

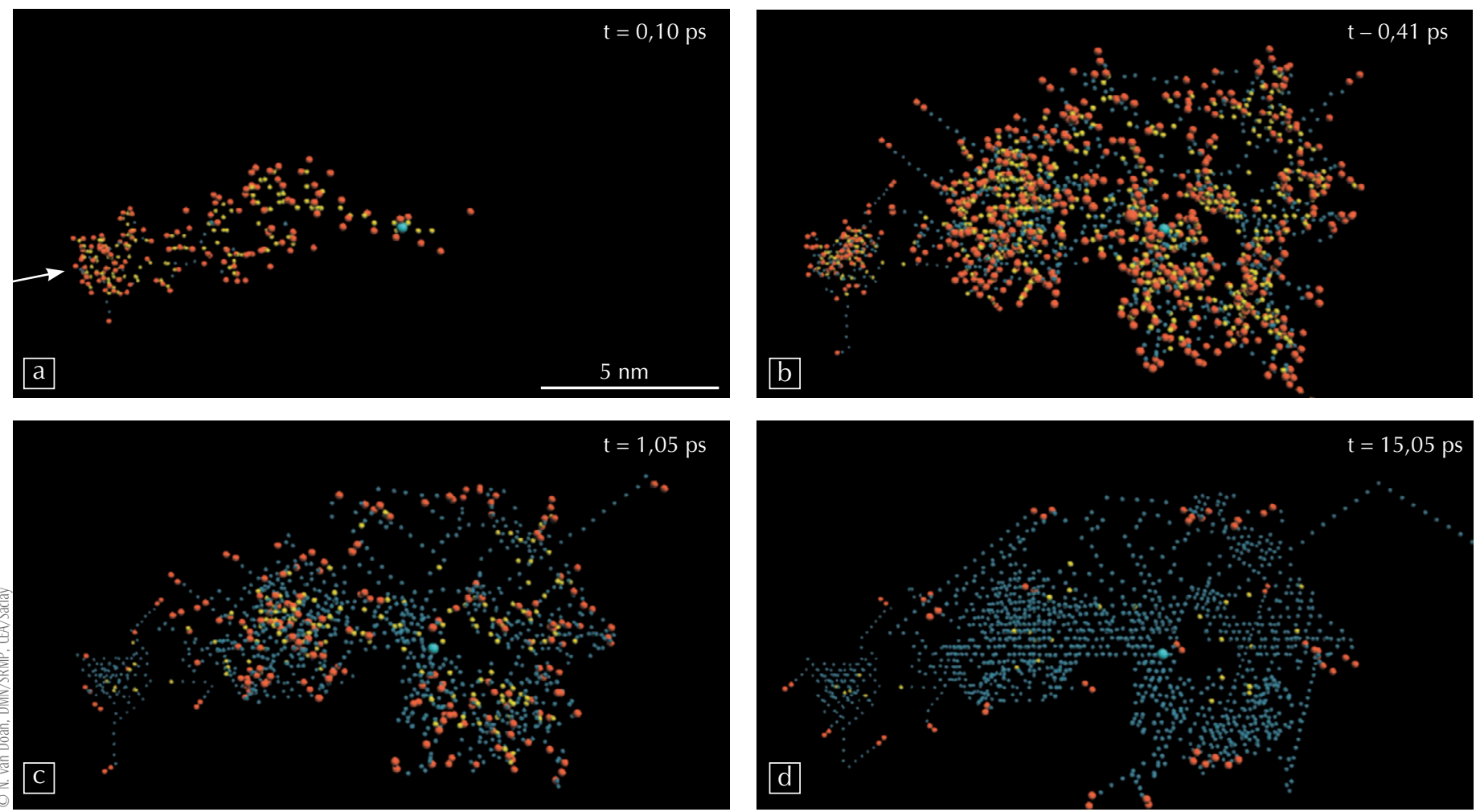

2. Évolution d'une cascade de déplacements atomiques due à un PKA de $10 \mathrm{keV}$ dans un alliage fer-1\%cuivre, en fonction du temps en picosecondes. Les lacunes sont en jaune, les interstitiels en rouge et les atomes remplacés en bleu. L'atome plus gros en bleu clair est le PKA, qui arrive dans la direction indiquée par une flèche et est thermalisé après 0,1 ps. Le calcul est fait par dynamique moléculaire, où l'on modélise en mécanique classique le mouvement des atomes individuels en supposant que les forces entre ceux-ci dérivent d'un potentiel fonction de la position des atomes. Dans un premier temps (0,10 et 0,41 ps après le choc, images a et b), on observe la formation de la cascade. Entre 1 et 15 ps (images c et d), la majorité des interstitiels se recombinent avec des lacunes. Dans l'image d, la plupart des lacunes et des interstitiels se sont recombinés. Les atomes affectés par la cascade sont en grande majorité des atomes remplacés, c'est-à-dire des atomes déplacés qui ont retrouvé un site cristallin vacant. Ne contribuant pas aux déplacements atomiques, ils sont sans effet sur des alliages désordonnés, mais peuvent être déterminants dans le cas de composés définis, par le désordre balistique qu'ils apportent.

\section{\〉}

\section{Transmutations : production d'hélium et d'hydrogène, et activation nucléaire}

Les réactions de transmutation nucléaire, qui se produisent lors de la collision initiale d'un neutron avec un atome du matériau cible, modifient la composition chimique. Celles produisant des particules $\alpha$ et des protons ont particulièrement retenu l'attention, car il s'agit des noyaux d'éléments (l'hélium et l'hydrogène) pouvant fragiliser les matériaux. Dans un spectre de réacteur de fusion, on produit typiquement $\sim 12$ appmHe/dpa (12 10-6 atomes d'hélium par atome déplacé) et $\sim 45 \mathrm{appmH} /$ dpa dans les alliages à base de fer que sont les aciers. Les sections efficaces (autrement dit les probabilités de production) ont tendance à diminuer quand la masse atomique croît : la production d'hélium dans le tungstène n'est que de $\sim 0,3 \mathrm{appmHe} / \mathrm{dpa}$, alors que dans un composé d'éléments légers, comme le carbure de silicium, on peut produire jusqu'à $110 \mathrm{appmHe/dpa} \mathrm{en}$ spectre de neutrons de fusion [3].

Cette production d'hélium et d'hydrogène est typique des neutrons de fusion de $14 \mathrm{MeV}$. Elle est de plusieurs ordres de grandeur supérieure à celle induite par les neutrons rapides de fission. Pour ces derniers, d'énergie plus faible, la production d'hélium dans le fer est de quelques 0,1 appm/dpa.

Les réactions de transmutation produisent aussi des isotopes radioactifs, qui ont pour conséquence une activation nucléaire des matériaux. Un acier martensitique conventionnel (voir encadré), de composition chimique Fe-9\%Cr-1\%Mo-V-Nb, émettra après une irradiation de $\sim 100 \mathrm{dpa}$ dans un réacteur de fusion un fort rayonnement gamma qui le fera considérer comme déchet de haute activité, jusqu'à $\sim 10^{5}$ ans après son emploi.
Cette radioactivité dépend de la composition chimique initiale. On a su fabriquer des aciers martensitiques à $9 \%$ de chrome à activation nucléaire réduite, comme l'EUROFER, sans dégrader les propriétés d'emploi, où l'on a remplacé le molybdène et le niobium (éléments qui s'activent fortement) par du tungstène, et limité les teneurs résiduelles en $\mathrm{Nb}, \mathrm{Mo}, \mathrm{Ni}, \mathrm{Cu}, \mathrm{Al}$ et $\mathrm{Co}$ : après quelques centaines d'années, son rayonnement gamma est de plusieurs ordres de grandeur inférieur à celui d'un acier conventionnel. Moyennant un contrôle plus strict de ces éléments, qui ne semble pas hors de portée de l'industrie sidérurgique, un acier EUROFER à basse activation nucléaire pourrait, après quelques centaines d'années de désactivation, être réintroduit sans précaution particulière dans le cycle de production [4]. 


\section{Gonflement : exemple des aciers austénitiques et ferritiques}

Le gonflement est une augmentation de volume, induite par l'agglomération sous forme de cavités des lacunes formées pendant l'irradiation. C'est un facteur limitant la durée d'utilisation du matériau dans le réacteur, par suite des modifications de propriétés mécaniques et dimensionnelles induites. Les différents aciers (voir encadré, p. 21), irradiés par des neutrons rapides issus de la fission, ont des comportements radicalement différents.

Le gonflement des aciers austénitiques est un phénomène à seuil, caractérisé par l'existence de deux régimes consécutifs : un premier régime, dit d'incubation, pendant lequel le gonflement est négligeable. Puis, au-delà d'une dose critique, dite dose d'incubation, une vitesse de gonflement stationnaire, de quelques 0,1 à $1 \%$ par dpa apparait (fig. 3a). La dose d'incubation dépend fortement de la composition chimique. Le gonflement induisant une fragilisation supplémentaire inacceptable au-delà de $\sim 2 \%$ de déformation, la fin de la période d'incubation signifie la fin de vie pour les aciers austénitiques. Ces aciers ne sont donc pas envisagés comme matériaux de structure de la première paroi des futurs réacteurs de fusion, pour laquelle on prévoit des doses correspondant à 50-80 dpa.

À l'opposé, les aciers ferritiques et martensitiques irradiés par des neutrons de fission ont une excellente résistance au gonflement pour les doses explorées inférieures à $\sim 150 \mathrm{dpa}$. Sous irradiation par des ions produisant uniquement des déplacements atomiques (pas de réactions nucléaires), cette excellente résistance est confirmée. Mais sous irradiation en triple faisceau (création de déplacements atomiques par irradiation aux ions de fer, avec implantation simultanée d'hydrogène et d'hélium) simulant les conditions d'un réacteur de fusion, cette résistance se dégrade très sensiblement (fig. 3b). Il est donc probable qu'à cause du gonflement, l'EUROFER ait, dans les futurs réacteurs de fusion, une dose de fin de vie limitée à des valeurs significativement inférieures à celles que l'on pouvait déduire des irradiations par des neutrons rapides de fission. En revanche, dans des conditions similaires de triple faisceau, un acier ferritique renforcé par dispersion de particules d'oxyde (voir encadré, p. 21) montre une bien meilleure résistance au gonflement (fig. 3c).
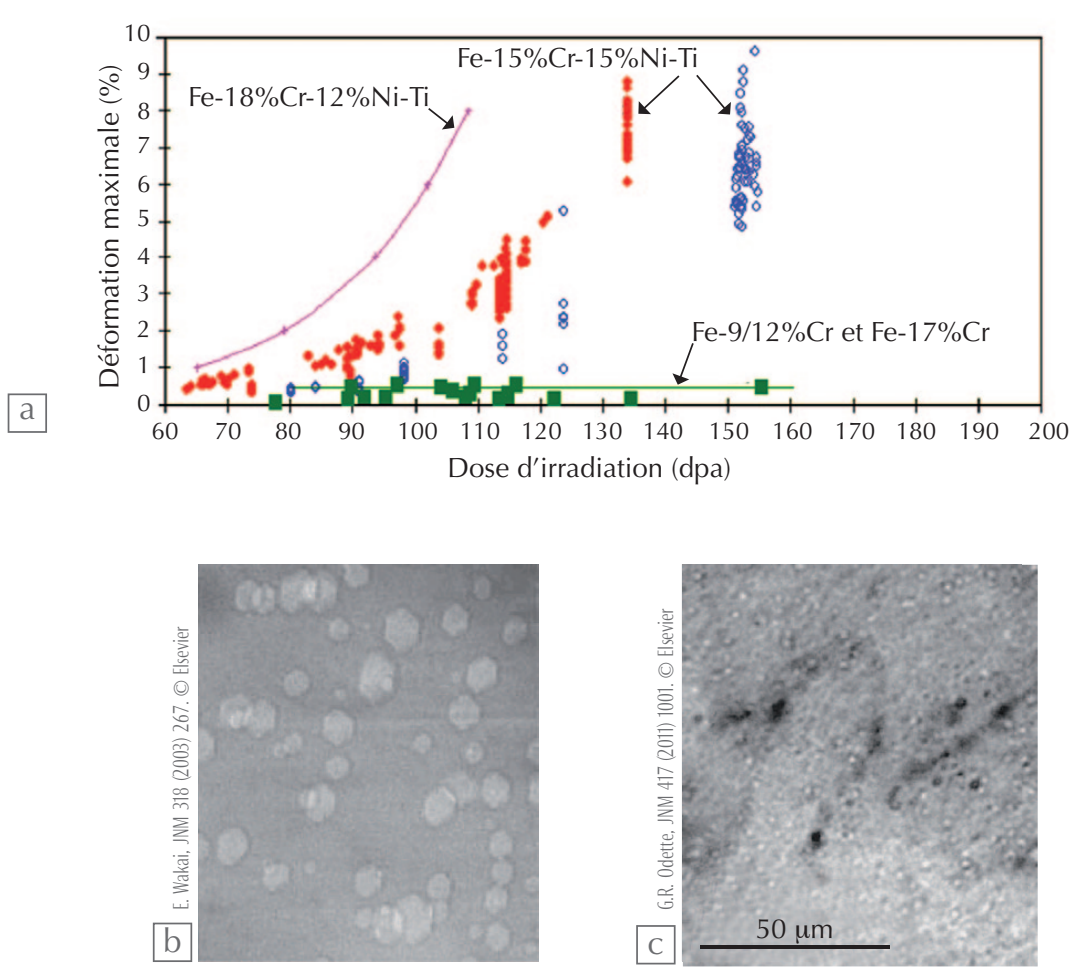

3. Gonflement des aciers sous irradiation.

(a) Déformation de gonflement en fonction de la dose pour des aciers austénitiques à 18\%Cr-12\%Ni-Ti (courbe rose) et 15\%Cr-15\%Ni-Ti (cercles rouges et bleus), et des aciers martensitiques à 9-12\%Cr et ferritiques à 17\%Cr (carrés verts), irradiés aux neutrons rapides dans le réacteur Phénix (d’après J.L. Séran, communication personnelle). (b) Micrographie électronique en transmission d'un acier martensitique à $9 \% \mathrm{Cr}$ à activation réduite, irradié à $470^{\circ} \mathrm{C}$ sous triple faisceau d'ions simulant des conditions typiques de la fusion : 50 dpa, 900 appm He et 3500 apmm H [7] : on observe des cavités, responsables d'un gonflement significatif.

(c) Acier ferritique $0 D S$ à $14 \%$ Cr irradié à $500^{\circ} \mathrm{C}$ jusqu'à 9 dpa, avec production simultanée de 380 appm He [8] : le gonflement est négligeable.

\section{Durcissement et fragilisation des aciers ferritiques et martensitiques}

Les propriétés mécaniques sont particulièrement importantes pour les matériaux de structure. Les principales sont la limite d'élasticité, contrainte appliquée au-dessus de laquelle le matériau devient plastique, et la déformation à rupture (la ductilité) ; ces quantités sont déterminées par essai de traction (mesure de la contrainte en fonction de la déformation, à vitesse de déformation imposée). Dans les essais de fluage, on mesure la (lente) déformation d'un matériau soumis à une contrainte constante. La résistance à la propagation de fissures (la ténacité) est évaluée en soumettant des éprouvettes pré-fissurées à des essais de traction.

Les métaux peuvent rompre de manière fragile par clivage, c'est-à-dire par séparation de deux plans cristallins sans déformation plastique macroscopique, ou ductile, après déformation plastique significative. La contrainte de clivage ne dépend pas de la température. Pour les métaux cubiques centrés, la mobilité des dislocations est thermiquement activée et la limite élastique décroit avec la température. Il en résulte une température de transition fragile-ductile (TTFD), entre régime fragile à basse température et régime ductile à haute température (fig. 4a).

Pour des températures inférieures à la TTFD, l'énergie nécessaire à la propagation d'une fissure et la ténacité sont faibles : on parle de rupture fragile. Au-dessus de la TTFD, la forte déformation plastique nécessaire à la propagation d'une fissure conduit à des énergies à rupture et des ténacités très élevées. Pour éviter de se trouver dans le domaine de rupture fragile, la bonne pratique de l'ingénieur veut que les matériaux de structure sélectionnés gardent, en toute circonstance, une température de transition inférieure aux températures de service et de manutention.

\> 


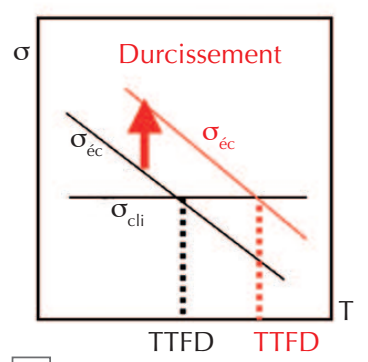

$\mathrm{a}$
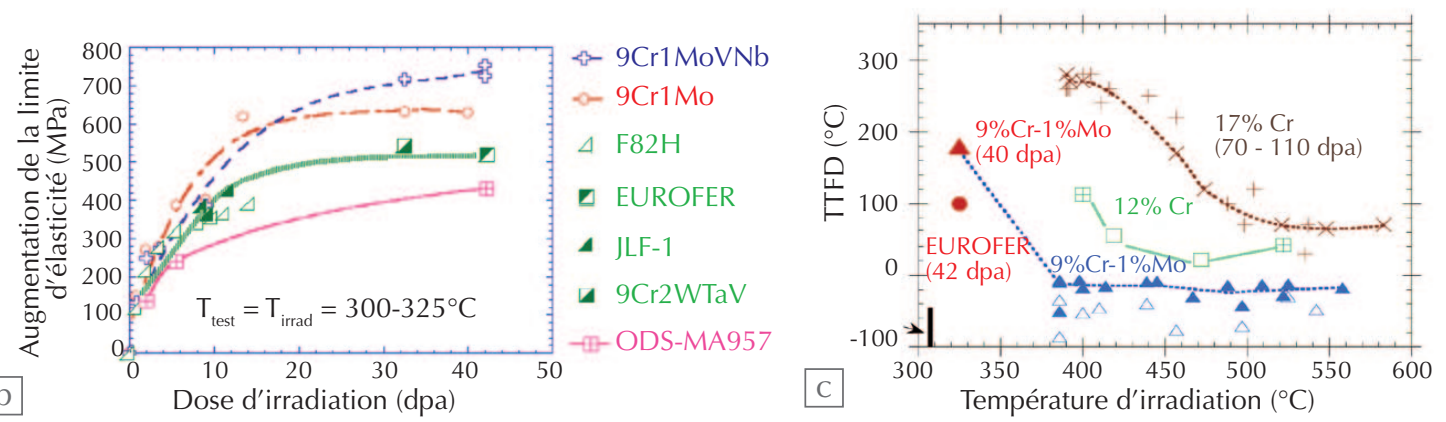

4. Durcissement et fragilisation des aciers ferritiques et martensitiques irradiés aux neutrons rapides.

(a) Définition de la température de transition fragile-ductile TTFD. Un durcissement du matériau se traduit par une augmentation de la contrainte d'écoulement plastique $\sigma_{\hat{e} e}$ et donc de TFD. II n'affecte pas la contrainte de clivage $\sigma_{\text {cli }}$.

(b) Durcissement de divers aciers martensitiques en fonction de la dose d'irradiation (en dpa), mesuré par l'augmentation de la limite d'élasticité à la température d'irradiation $\left(\sim 325^{\circ}\right)$. Les symboles de couleur verte regroupent les aciers martensitiques à basse activation. (A. Alamo, J.Nucl. Mater. 367-370 (2007) 54-59).

(c) Température de transition fragile-ductile TFD d'aciers martensitiques et ferritiques, après irradiation en réacteur à neutrons rapides, en fonction de la température d'irradiation. La TTFD des aciers non irradiés se situe entre - 100 et -50 ${ }^{\circ}$ C. (J.L. Boutard, C.R Physique 9 (2008) 287-302).

\section{\〉}

\section{Durcissement et fragilisation} par les neutrons rapides de fission

Les amas de défauts ponctuels (résidus des cascades de déplacements) créés par l'irradiation aux neutrons rapides sont des obstacles au glissement des dislocations. Ils conduisent donc à un durcissement (augmentation de la contrainte d'écoulement plastique), et par conséquent à une augmentation de la TTFD (fig. 4a), ce qui risque de la faire passer au-dessus de la température de service. Il y a alors danger de fragilisation du matériau en conditions de service. Ce durcissement est d'autant plus important que la température d'irradiation est basse, et dépend aussi de la composition et de la microstructure de l'acier.

Par exemple, l'irradiation par neutrons rapides de fission à $\sim 325^{\circ} \mathrm{C}$ durcit les aciers martensitiques (fig. 4b) et augmente significativement les températures de transition fragile-ductile de l'EUROFER et d'un acier conventionnel (9\% Cr-1\%Mo) (fig. $4 \mathrm{c}$ ). En revanche, irradiés à plus haute température, entre 400 et $550^{\circ} \mathrm{C}$, ces mêmes aciers martensitiques à $9 \%$ de chrome ne sont que marginalement fragilisés (fig. 4c, triangles bleus) et durcis. Cette fenêtre de température d'irradiation $\left(400-550^{\circ} \mathrm{C}\right)$ est donc optimale pour la bonne tenue mécanique de ces aciers dans les réacteurs de fission. La figure 4c montre aussi l'influence flagrante de la teneur en chrome : irradiés dans les mêmes conditions de température $\left(400-550^{\circ} \mathrm{C}\right)$, les aciers ferritiques à $17 \% \mathrm{Cr}$ présentent une forte fragilisation associée à un fort durcissement, induits en grande partie par une accélération de la décomposition de phase $\alpha / \alpha^{\prime}$ (voir encadré, p. 21).

\section{Fragilisation par l'hélium}

En l'absence d'une source de neutrons de $14 \mathrm{MeV}$, l'effet de l'hélium qu'ils produiraient dans un réacteur de fusion a été simulé par différentes méthodes, dont l'implantation de particules $\alpha$ d'énergie de $\sim 25 \mathrm{MeV}$. Les défauts créés par les particules $\alpha$ sont relativement peu nombreux; il en résulte des rapports $\mathrm{He} / \mathrm{dpa}$ très élevés, $\sim 10^{4} \mathrm{appm} / \mathrm{dpa}$. L'implantation d'hélium à $250^{\circ} \mathrm{C}$ induit un fort durcissement (fig. 5a) et l'apparition d'une rupture intergranulaire fragile. Cette dégradation est associée à une microstructure très dense de bulles d'hélium (fig. 5b). À durcissement équivalent obtenu après irradiation par les neutrons rapides d'un réacteur de fission (sans production d'hélium), le même acier rompt de manière transgranulaire ductile, ce qui conduit à penser que l'hélium implanté fragilise au-delà du durcissement. Bien qu'aucune bulle d'hélium n'ait pu être observée aux joints de grains dans les échantillons implantés à $250^{\circ} \mathrm{C}$, l'hypothèse raisonnable est que l'hélium à l'état d'atomes ou d'amas nanométriques puisse abaisser l'énergie de cohésion des joints de grains [9]. Pour des joints de géométrie simple, cette hypothèse est en accord qualitatif avec des résultats récents de calculs ab initio d'énergie de cohésion dans le fer [10].

Ces implantations sont des simulations un peu extrêmes. Néanmoins, l'analyse des résultats de fragilisation après irradiation dans différents spectres de neutrons permettant de faire varier le rapport $\mathrm{He} / \mathrm{dpa}$, montre que, très vraisemblablement cette fragilité intergranulaire de basse température est à craindre pour les aciers martensitiques à
9\%Cr à partir de 500 appm d’hélium [11], soit $\sim 50$ dpa en spectre de fusion (neutrons de $14 \mathrm{MeV})$. De plus, en conditions de fluage vers $550^{\circ} \mathrm{C}$, après implantation de particules $\alpha$, on observe des bulles d'hélium situées aux joints de grains (fig. 5c) qui croissent par absorption de lacunes et finissent par entrer dans un régime de croissance instable provoquant des ruptures intergranulaires prématurées [12].

Un objectif important d'un programme de développement de matériaux pour un futur réacteur de fusion sera de retarder ou de s'affranchir de cette fragilisation par l'hélium. C'est l'objectif de la nanostructuration des aciers par dispersion d'oxyde que de piéger l'hélium au sein des grains, afin qu'il ne fragilise pas les joints (voir ce numéro, p. 24).

\section{Conclusion}

Les matériaux de structure qui seront soumis à de fortes doses d'irradiation (> $50 \mathrm{dpa}$ ) dans les futurs réacteurs de fusion, devront montrer un faible gonflement sous irradiation, rester dans le domaine ductile en conditions de service et éviter la fragilisation par l'hélium, avoir une résistance mécanique suffisante à haute température, et pouvoir subir des flux thermiques significatifs. Les aciers austénitiques sont exclus pour de telles applications. Nous avons vu que les aciers martensitiques, ceux renforcés par dispersion de particules d'oxydes, et leur versions à basse activation nucléaire, semblent des voies particulièrement prometteuses.

Disposer de matériaux capables de résister aux conditions de service des réacteurs de 

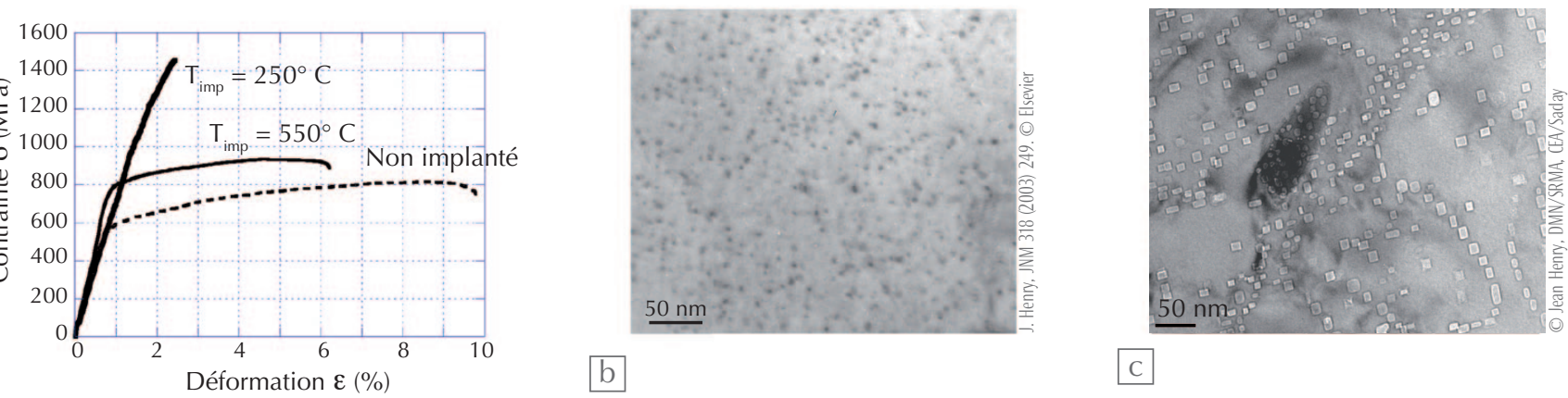

b

5. Effet de l'implantation d'hélium sur les propriétés mécaniques et la microstructure d'aciers martensitiques.

(a) Courbes contrainte-déformation, mesurées en essai de traction à la température ambiante, après implantation de 5000 appm d'hélium à $250^{\circ} \mathrm{C}$ (rupture fragile intergranulaire) et $550^{\circ} \mathrm{C}$ (rupture ductile transgranulaire).

(b) Microstructure dense de bulles d'hélium (petites taches blanches) après implantation à $250^{\circ} \mathrm{C}$, observée par microscopie électronique en transmission (MET) [9].

(c) Microstructure de bulles d'hélium (petites taches blanches) situées préférentiellement aux joints de grains ou près des dislocations, après implantation à $550^{\circ} \mathrm{C}$, observée en MET.

fusion constitue aujourd'hui un verrou dans le développement de ce type de réacteur. Le second article de ce dossier présentera l'effort de recherche, développement et qualification de matériaux de structure et/ ou de protection face au plasma thermonucléaire, capables de résister aux forts flux de neutrons de $14 \mathrm{MeV}$ mais aussi, en ce qui concerne les matériaux de protection, à de forts flux thermiques et de particules.

(a) La transmutation d'un élément correspond à la modification du nombre de neutrons et de protons de son noyau à la suite de réactions nucléaires. Très souvent le nombre de protons est affecté, donc la nature chimique de l'élément, dont le noyau subit une transmutation, est aussi changée.

Remerciements

Je voudrais remercier mes collègues du CEA pour leur relecture et commentaires critiques : Jean Henry et François Willaime, du Département des Matériaux pour le Nucléaire.

\section{Références}

1- C. Lemaignan, Science des matériaux pour le nucléaire, EDP Sciences (2004).

2- D. N. Seidman et al., Nuclear Instruments and Methods, 182-183 (1981) 477-481.

3• M. R. Gilbert et al., J. Nud. Mater., 442 (2013) S755-S760.

4• R. Lindau et al., Fusion Eng. and Des., 75-79 (2005) 989-996.

5• J. Philibert et al., Métallurgie : du minerai au matériau, $2^{\mathrm{e}}$ édition, Dunod (2013).

6• Y. de Carlan, Clés CEA, 59 (2010), pp. 31-34.

7• E. Wakai et al., J. Nucl. Mater., 318 (2003) 267-273.

8• G. R. Odette et al., J. Nucl. Mater., 417 (2011) 1001-1004.

9• J. Henry et al., J. Nucl. Mater., 318 (2003) 249-259.

10• L. Zhang et al., Phys. Rev. B, 87 (2013) 134107

11• T. Yamamoto et al., J. Nucl. Mater., 356 (2006) 27-49.

12• H. Ullmaier et al., Materials Science Forum, 97-99 (1992) 451.

\section{Les aciers utilisés dans les réacteurs nucléaires [5]}

Les aciers sont des alliages à base de fer, contenant $0,02 \%$ à $2 \%$ de carbone en poids et d'autres éléments. Le fer présente deux formes, de structures cristallines cubique centrée (phase $\alpha$, stable en dessous de $912^{\circ} \mathrm{C}$, et phase $\delta$, stable de $1394^{\circ} \mathrm{C}$ à la température de fusion) et cubique à faces centrées (phase $\gamma$, stable entre $912^{\circ} \mathrm{C}$ et $1394^{\circ} \mathrm{C}$ ). Des éléments d'alliage peuvent stabiliser l'une ou l'autre de ces structures cristallines : par exemple, le chrome et le molybdène stabilisent la phase $\alpha$, ou ferrite ; le nickel et le carbone la phase $\gamma$, ou austénite.

Les aciers austénitiques, qui constituent la majorité des aciers inoxydables, sont caractérisés par une structure cristalline cubique à faces centrées. Ils contiennent typiquement 17 à $18 \%$ de chrome, 8 à $12 \%$ de nickel, moins de $0,1 \%$ de carbone et divers éléments mineurs ( $\mathrm{Si}, \mathrm{Mo}, \mathrm{Ti}, \mathrm{Nb} . .$. pour améliorer leurs propriétés. Ils ont une ductilité et une ténacité remarquables, associées à une bonne tenue en fluage thermique mais, pour le nucléaire, ils présentent l'inconvénient d'un gonflement excessif sous irradiation. Par ailleurs, comparés aux aciers ferritiques ou martensitiques, ils ont des conductivités thermiques relativement moyennes associées à des coefficients de dilatation élevés, ce qui induit des contraintes mécaniques d'origine thermique plus élevées en cas de transfert de flux de chaleur, ce qui est défavorable.

Les aciers ferritiques présentent une structure cristalline cubique centrée jusqu'au point de fusion. Ils ont une faible teneur en carbone, une concentration élevée de chrome (12 à 28\%), pas de nickel, et $\sim 1 \%$ de molybdène. Dans le fer, le chrome présente une limite de solubilité d'environ $9 \%$, au-delà de laquelle la solution solide $\mathrm{Fe}-\mathrm{Cr}$ métastable se décompose progressivement en une phase $\alpha$ riche en fer et une phase $\alpha^{\prime}$ riche en chrome, ce qui se traduit par un durcissement.

Les aciers martensitiques sont obtenus par refroidissement rapide depuis la phase austénitique de haute température, suivi d'un traitement thermique dit de revenu vers $700^{\circ} \mathrm{C}$. Ceci leur donne une structure cristalline cubique centrée et une microstructure martensitique en forme d'aiguilles ou de lattes, à l'origine de propriétés mécaniques élevées, qui peuvent être optimisées par la durée et la température du traitement final de revenu. Ils contiennent typiquement 9 à $12 \%$ de chrome, 0,08 à $0,15 \%$ de carbone, ainsi que divers éléments d'addition (Mo, Nb, remplacés par $\mathrm{W}$ pour les alliages à faible activation).

Les aciers martensitiques et ferritiques sont meilleurs conducteurs de la chaleur que les aciers austénitiques avec un coefficient de dilatation plus faible, ce qui est favorable pour transmettre des flux de chaleur importants. Leur résistance au gonflement est excellente. Cependant leur résistance au fluage devient trop faible au-delà de $550^{\circ} \mathrm{C}$ pour des applications structurelles. Les aciers ODS (Oxide Dispersion Strengthened) ont été développés afin d'améliorer sensiblement les résistances des aciers ferritiques ou martensitiques au fluage à haute température [6], et au gonflement sous irradiation en réacteur de fusion (voir p. 19). Ils sont ferritiques ou martensitiques, en fonction de leur teneur en chrome, et renforcés par une dispersion dense de 0,2 à $0,6 \%$ en volume, de particules d'oxydes d'yttrium ou de titane, de dimensions nanométriques. 